\title{
La salud mental en primera persona. Los comités de personas expertas
}

\section{First-person perspective on mental health. Committees of expert people}

\section{Palabras clave:}

Salud mental, participación, empoderamiento, comités, Salud Mental España.

\section{Keywords:}

Mental health, participation, empowerment, committees, Mental Health Spain.

\section{Introducción}

Existen múltiples barreras que obstaculizan el derecho a la participación de las personas con problemas de salud mental, como los prejuicios, la discriminación, las etiquetas, el paternalismo, la falta de información y formación, el tradicionalismo o el escepticismo. El hecho de poder participar en la toma de decisiones ofrece multitud de ventajas tanto desde la perspectiva de derechos como para el empoderamiento y la recuperación.

La participación es un derecho reconocido. Aunque lamentablemente la participación en salud mental es aún escasa, desde Salud Mental España se considera imprescindible fomentar la implicación real y efectiva de todas las personas que se pueden ver, en algún momento de su vida, afectadas por problemas de salud mental.

La Confederación Salud Mental España ${ }^{\mathrm{I}}$ es una entidad sin ánimo de lucro de interés social y declarada de utilidad pública que nació en I983 y cuya misión es mejorar la calidad de

I. En 2015 la Confederación Feafes actualizó su marca y pasó a denominarse Salud Mental España.

\section{Salud Mental España}

<confederacion@consaludmental. org >

Para citar:

Salud Mental España (20I6): "La salud mental en primera persona. Los comités de personas expertas", Revista Española de Discapacidad, 4 (I): $24 \mathrm{I}-247$.

Doi: <http://dx.doi.org/I0.5569/23405IO4.04.OI.I4> 
vida, defender los derechos de las personas con enfermedad mental y sus familias y representar a este movimiento asociativo. En la actualidad, integra a I9 federaciones autonómicas y asociaciones uniprovinciales, que a su vez agrupan a casi 300 asociaciones y suman más de 44.000 socios y socias en todo el territorio estatal.

Según la Guía Partisam², una herramienta para el fomento de la participación de las personas usuarias de los servicios de salud mental coordinada por la Confederación y editada por la Asociación Española de Neuropsiquiatría (AEN), la participación "supone una toma de poder personal, que capacita a la persona para tener el control sobre su propia vida. Es decir, facilitar el empoderamiento de las personas con problemas de salud mental, así como de sus familias como personas cuidadoras" (Fernández de Sevilla y San Pío, 20I4).

La Organización Mundial de la Salud (OMS) considera que el "empoderamiento" es un concepto esencial de la promoción de la salud. Ya desde la Declaración de Alma-Ata (OMS, I978) se reconoce que "las personas tienen el derecho y el deber de participar individual y colectivamente en la planificación e implementación de su atención sanitaria”.

Aplicado a las personas con problemas de salud mental, la OMS indica que "en el contexto de la salud mental, el empoderamiento se refiere al nivel de elección, influencia y control que los usuarios/as de estos servicios ejercen en las situaciones que acontecen en sus vidas" (OMS, 20IO). Es por ello que es tan importante facilitar que estas personas tomen las riendas y encabecen las iniciativas, propuestas y acciones que se lleven a cabo en todos los aspectos relacionados con la defensa de sus derechos y su calidad de vida y bienestar.

2. "Guía Partisam. Promoción de la participación y autonomía en salud mental", texto disponible en <https://consaludmental. org/centro-documentacion/promocionautonomia/guia-partisam-20686/> [consulta: I de marzo de 20I6].

\section{“¿Qué es el empoderamiento?” Decálogo “¿Qué es el empoderamiento?” (2014). Comité Pro Salud Mental en Primera Persona de Salud Mental España}

1. Plenitud. La existencia de las personas tiene múltiples facetas, y queremos disfrutar de todas ellas.

2. Control. Queremos ser los protagonistas de nuestras vivencias.

3. Superación. Queremos potenciar nuestras habilidades.

4. Responsabilidad. Queremos poner en valor nuestra madurez, sin paternalismos.

5. Autonomía. Queremos cerrar los círculos del trabajo y la afectividad.

6. Bienestar. Tanto social como individual; queremos ser felices.

7. Autoconocimiento. Debemos aceptar con humildad nuestras limitaciones para evitar frustraciones innecesarias.

8. Confianza. Al mismo tiempo debemos trasmitir seguridad a la hora de relacionarnos, sin complejos.

9. Autoestima. (De gran valor terapéutico).

Io. Participación. "Nada de nosotros sin nosotros".

El estigma es una de las barreras invisibles fundamentales -si no la más importante-que las personas con problemas de salud mental tienen que superar para lograr que su proceso de recuperación sea posible (Red2Red, 20I 5 ). Una de las mejores maneras de combatirlo es conociendo de primera mano a las personas que han tenido o tienen estos problemas, escuchando sus voces y sus experiencias. Es así como se convierte el "los otros" en "nosotros", ya que son más las cosas que tenemos en común que las que nos separan, y no sólo porque una de cada cuatro personas $^{3}$ pueda llegar a desarrollar un problema de salud mental a lo largo de su vida, sino porque todos, como ciudadanos, tenemos los mismos derechos y obligaciones.

3. Según datos de la Estrategia I de cada 4, enmarcada en el Plan Integral de Salud Mental de la Consejería de Salud de Andalucía. Accesible en: <www.Idecada4.es> [consulta: I 5 de febrero 2016]. 


\section{Qué es el Comité Pro Salud Mental en Primera Persona ${ }^{4}$}

Para la OMS, "la clave del empoderamiento es la eliminación de los impedimentos formales e informales, así como la transformación de las relaciones de poder entre individuos, comunidades, servicios y gobiernos" (OMS, 20IO). Salud Mental España, consciente de ello, ha querido transformar esas relaciones impulsando desde 2009 la creación de comités formados por personas con experiencia propia en salud mental.

Estos comités están formados por personas con problemas de salud mental que participan activamente en la vida de sus asociaciones y que creen en la máxima "nada sobre nosotros sin nosotros", partiendo de la base de que la propia experiencia aporta un punto de vista fundamental a la hora de analizar y defender los derechos de este colectivo y sus familias.

Con la creación de los comités se materializa el fuerte compromiso de los órganos de gobierno de la Confederación por promover y facilitar la participación real de las personas con problemas de salud mental en su funcionamiento y toma de decisiones.

Existe un comité a nivel estatal, compuesto por representantes de cada una de las entidades autonómicas miembro de la Confederación. A su vez, estas organizaciones han ido creando progresivamente sus propios comités a nivel autonómico.

El Comité Pro Salud Mental en Primera Persona asesora a los órganos de gobierno ${ }^{5}$, desarrolla propuestas, propone proyectos, establece posicionamientos y da voz a las personas con trastorno mental tanto de cara a la organización como a la sociedad en general.

4. Hasta marzo de 2016 este comité se denominaba "Comité de Personas con Enfermedad Mental”.

5. Estructura Organizativa de Salud Mental España, disponible en <https://consaludmental.org/estructura-organizativa I > [consulta: I 5 de febrero 2016].
Entre otras, sus funciones son debatir y estudiar asuntos de interés para las personas con estos problemas de salud y fijar posturas al respecto, potenciar la inserción laboral, intercambiar experiencias y buenas prácticas desarrolladas en las comunidades autónomas, luchar contra el estigma y elaborar una agenda de reivindicaciones y estrategias a seguir.

En línea con la filosofía tanto del Comité como de la propia Confederación de ofrecer una visión positiva de la salud mental, en su última reunión los miembros del Comité decidieron cambiar su nombre, sustituyendo "enfermedad mental" por una expresión que pusiera el énfasis tanto en la experiencia propia de la persona como en el concepto de salud más que en el de enfermedad, pasando a denominarse Comité Pro Salud Mental en Primera Persona.

\subsection{Objetivos, reglamento y organización}

Según el reglamento interno elaborado por el propio Comité y que fue aprobado por la Junta Directiva en 20I0, para formar parte del Comité es necesario que exista una designación formal por parte de las entidades autonómicas. Actualmente, salvo una, existe representación de todas las autonomías ${ }^{6}$.

Para desarrollar sus funciones, el Comité se reúne de forma presencial dos veces al año, y de forma no presencial, tantas veces como sean necesarias. En las reuniones se toman las decisiones por consenso y, si no hay acuerdo, el reglamento contempla que se adopten por mayoría simple.

\subsection{Un poco de historia: los inicios del Comité Pro Salud Mental en Primera Persona \\ El 20 de enero de 2009 se organizó un grupo de trabajo en el que se debatió la participación de las personas con trastorno mental en el}

6. Comités y comisiones de la Confederación Salud Mental España, disponibles en <https://consaludmental.org/estructuraorganizativa I> [consulta: I de marzo 20I6]. 
movimiento asociativo agrupado en Salud Mental España.

En este debate, los I 5 asistentes mostraron su opinión acerca de qué es participación, cuál era su experiencia participativa en su propia asociación o federación, las dificultades que se encontraban para participar y cuáles eran los aspectos que podrían favorecerla.

El acta de dicha reunión, con todas las propuestas y conclusiones, se hizo llegar a la Comisión Permanente de la Confederación que, tras la lectura atenta de la misma, propuso finalmente la creación de un Comité Asesor de Personas con Enfermedad Mental, iniciativa que se aprobó por la Junta Directiva el 2 I de febrero de 2009. Algunos participantes en el primer grupo de trabajo intervinieron en las XVI Jornadas de Feafes, celebradas en Cuenca del 22 al 24 de octubre.

Una vez culminado el proceso de designación de representantes autonómicos, el 2I de septiembre de 20 Io se celebró en la sede de Salud Mental España la primera reunión del recién constituido Comité de Personas con Enfermedad Mental que, como órgano asesor, formaba ya parte de la estructura de la Confederación.

A esta primera reunión acudieron doce representantes designados por nueve federaciones y asociaciones uniprovinciales integradas en la Confederación: Feafes Andalucía, Feafes Afesa Asturias, Febafem (Baleares), Ascasam (Cantabria), Feafes Castilla y León (actualmente Federación Salud Mental Castilla y León), Fecafamm de Cataluña (actualmente Federació Salut Mental Catalunya), Feafes Extremadura (actualmente Feafes Extremadura Salud Mental), Anasaps (Navarra), Acefep de Ceuta (actualmente Asociación Salud Mental Ceuta) y un invitado por la, entonces, Confederación Feafes.

En este encuentro, se designó al representante de Ceuta, Basilio García Copín, como responsable del Comité y a la representante de Castilla y León, Elena Briongos Rica, como secretaria.
En la Junta Directiva de la Confederación del I I de diciembre de 20 Io finalmente se aprobó, por unanimidad, el reglamento de régimen interno del Comité.

Entre otras actividades, descritas posteriormente, miembros del Comité participaron en la elaboración del Plan Estratégico 20I 2-20I6 de Salud Mental España. Tal y como se indica en este Plan Estratégico (Feafes, 20I2), la participación de las personas con problemas de salud mental es la base de una de las líneas estratégicas principales, referida a la incidencia social y política de la Confederación? 7 .

\subsection{Un poco de historia: un recorrido por las principales actividades realizadas}

Desde que el Comité comenzara a andar, sus acciones han girado principalmente en torno a la defensa y ejercicio de los derechos de las personas con problemas de salud mental, la eliminación de barreras invisibles que dificultan la plena participación social y la puesta en marcha de estrategias con las que fomentar la creación de redes y el fortalecimiento del tejido asociativo.

El Congreso Feafes celebrado en Valladolid los días 5, 6 y 7 de mayo de 20 I I fue uno de los primeros grandes hitos para el Comité en tanto compartió con el movimiento asociativo su visión y propuestas en materia de empleo, implantación de la Estrategia en Salud Mental ${ }^{8}$ del Sistema Nacional de Salud (Gómez, 20II), y aplicación en España de la Convención de la ONU sobre los Derechos de las Personas con Discapacidad.

\footnotetext{
7. Línea Estratégica 3: Incidencia social y política. Objetivo Estratégico 5: Reforzar el trabajo para el empoderamiento de las personas con enfermedad mental, apoyándonos en la Convención de la ONU, el reconocimiento de sus derechos y el modelo de intervención comunitaria (Plan Estratégico 20I22016 de Salud Mental España).

8. Propuestas del Comité de Personas con enfermedad Mental para la Estrategia de Salud Mental, documento disponible en $<$ https://consaludmental.org/publicaciones/PropuestasEstrategiaSM-ComitePersonasconenfermedadmental.pdf >.
} 
En noviembre de ese mismo año y a petición de la Confederación, Elena Briongos comenzó a participar junto con el presidente de Salud Mental España, en el Comité de Seguimiento y Evaluación de la Estrategia en Salud Mental del Sistema Nacional de Salud para saldar en parte una deuda histórica e introducir así la voz de las personas con problemas de salud mental en el foro donde se estaban decidiendo las principales recomendaciones para la atención e intervención en materia de salud mental.

El Comité tomó parte activa de la campaña de la Confederación contra la propuesta de reforma del código penal, aportando ideas y estrategias para su diseño y desarrollo desde $20 \mathrm{I} 3 \mathrm{y}$ exponiendo sus propias razones contra esta reforma ${ }^{9}$, además de colaborar en la elaboración del folleto "Io motivos contra la reforma del Código Penal en materia de salud mental" y en el vídeo "Motivos no nos faltan" ${ }^{\circ}$. Esta campaña culminó en enero de 2015 , cuando se decidió en el Congreso retirar del Proyecto de Ley de modificación del Código Penal todas aquellas referencias a las medidas de seguridad de carácter 'indefinido' que se pretendían aplicar a las personas con trastorno mental.

Además de redactar y leer los manifiestos por el Día Mundial de la Salud Mental desde 20I $3{ }^{\text {II }}$, el Comité también ha asesorado a la Confederación, entre otras materias, sobre el enfoque y contenidos de proyectos ejecutados por Salud Mental España dirigidos al fomento

9. "Peligro, se legisla": las razones del Comité de Personas con Enfermedad Mental de Feafes en contra de la reforma del Código Penal. Disponible en <https://consaludmental.org/salaprensa/peligro-se-legisla-razones-comite-personas-enfermedadmental-feafes-contra-reforma-codigo-penal-I $7869 />$ [consulta: I 5 de febrero 20I6].

Io. "Motivos no nos faltan: Feafes contra la reforma del Código Penal", disponible en el canal de la Confederación Salud Mental España en youtube <https:/www.youtube.com/ watch?v=FWvMPyKeans $>$.

I I. Los manifiestos están disponibles en los siguientes enlaces: Manifiesto del Día Mundial de la Salud Mental 2OI 3: <https://consaludmental.org/general/manifiestodmsm-2013-2-I7500/>, Manifiesto Día Mundial de la Salud Mental 20I4: <http://www.atelsam.net/cms/wp-content/ uploads/Manifiesto-DMSM-2or4.pdf> y Manifiesto del Día Mundial de la Salud Mental 20I 5: <https://consaludmental. org/sala-prensa/manifiesto-del-dia-mundial-de-la-salud-mental-20I 5-2466o/s. de la participación social, el empoderamiento y el reconocimiento de derechos y sobre los elementos que deben componer los planes terapéuticos para las personas con problemas de salud mental.

Junto con las actividades que el Comité realiza como grupo asesor, sus miembros han colaborado en la elaboración de la Guía Partisam (Fernández de Sevilla y San Pío, 20I4), han actuado como facilitadores y formadores en diferentes programas formativos de la Confederación, desde 2014 asisten a reuniones de la Junta Directiva y forman parte del Consejo Consultivo de la Confederación creado en 2015.

\section{Los Comités autonómicos}

El desarrollo y establecimiento de comités en las diferentes comunidades autónomas es una apuesta firme y comprometida de los actuales órganos de gobierno de la Confederación por la participación directa, plena y activa de las personas con problemas de salud mental, que permite, además, reforzar las redes locales y fortalecer el movimiento asociativo.

Para Salud Mental España es muy importante generar estructuras que sirvan de ejemplo y modelo para ayudar a desmitificar los trastornos mentales y luchar contra el estigma. Es por ello que desde la Confederación se ha venido animando a las entidades a crear sus propios comités, con el objetivo de conseguir una estructura representativa y directa de los intereses concretos de las personas con problemas de salud mental.

A través del programa "Promoción de la Salud Mental y Prevención de la Exclusión”, financiado por el Ministerio de Sanidad, Servicios Sociales e Igualdad, desde 2013 se ha logrado consolidar la creación de estos comités. Esto, unido al compromiso de todas las entidades autonómicas, ha facilitado que actualmente un $84 \%$ de ellas cuenten ya con 
su propio comité de personas con experiencia propia en salud mental.

\section{Perspectivas de futuro}

Para los actuales órganos de gobierno de Salud Mental España, hacer valer el derecho a la participación de las personas con problemas de salud mental es una prioridad estratégica de máxima importancia que está naturalmente vinculada a la defensa de los derechos humanos y que a día de hoy se ha convertido en garantía fundamental para el adecuado desarrollo y sostenibilidad del movimiento asociativo del ámbito de la salud mental.

En este sentido, los miembros del Comité estatal ejercen habitualmente como portavoces ante los medios de comunicación, intervienen en jornadas y mesas redondas de todo el país y canalizan y dinamizan la información y el intercambio de experiencias desde y hacia las entidades autonómicas y la Confederación estatal.

Los órganos de gobierno seguirán promoviendo su participación en las actividades de la Confederación al entender que el Comité es una herramienta vital y de enorme trascendencia para que Salud Mental España pueda cumplir con la misión que el movimiento asociativo de familiares y personas con trastorno mental le ha encomendado y dar respuesta, además, a su compromiso estratégico sobre el fomento del empoderamiento y el logro de objetivos en cuestiones de incidencia social y política.

Además, tanto para el corto como el medio plazo, se está procurando la elaboración de proyectos y planes con los que hacer valer el derecho a la participación y gobierno de las personas con problemas de salud mental en el movimiento asociativo y en todas aquellas decisiones que les afectan y con los que fortalecer el apoyo mutuo e intercambio de experiencias y conocimiento. 


\section{Referencias bibliográficas}

Feafes (2012): Plan Estratégico 20I2-20I6, Madrid: Feafes (en línea). <https:// consaludmental.org/publicaciones/ PlanEstrategicoVersionResumida.pdf>, acceso 29 de abril de 2016 .

Feafes (20II): XVII Congreso FEAFES: el reto de la coordinación sociosanitaria, Valladolid: Feafes (en línea). <https://consaludmental.org/ centro-documentacion/xvii-congreso-feafesponencias-447I/>, acceso 29 de abril de 20 I 6.

Fernández de Sevilla, J. P. y San Pío, M. J. (coords.) et al. (20I4): Guía Partisam: promoción de la participación y autonomía en Salud Mental, Madrid: Asociación Española de Neuropsiquiatría (AEN) (en línea). <https:// consaludmental.org/publicaciones/PARTISAM. pdf $>$, acceso 29 de abril de 2016.

Gómez Beneyto, M. (coord.) et al. (2OI I): Estrategia en Salud Mental del Sistema Nacional de Salud 2009-2013, Madrid: Ministerio de Sanidad, Política Social e Igualdad (en línea). <https://consaludmental. org/centro-documentacion/estrategia-saludmental-2474/>, acceso 29 de abril de 2016 .
OMS (2010): Empoderamiento del usuario en salud mental, Madrid: Organización Mundial de la Salud Europa (OMS), Oficina Regional para Europa (en línea). <https://consaludmental.org/ publicaciones/Empoderamientosaludmental. pdf>, acceso 29 de abril de 2016.

OMS (I978): Declaración de Alma-Ata, URSS: Conferencia Internacional de Atención Primaria de Salud. OMS (en línea). <http:// www.alma-ata.es/declaraciondealmaata/ declaraciondealmaata.html>, acceso 29 de abril de 2016.

ONU (2006): Convención sobre los Derechos de las Personas con Discapacidad, ONU (en línea). <http://www.convenciondiscapacidad. es/ConvencionEspana_new.html>, acceso 29 de abril de 2016 .

Red2Red (2015): Salud mental e inclusión social: situación actual y recomendaciones contra el estigma, Madrid: Confederación Salud Mental España (en línea). <https://consaludmental.org/ publicaciones/Salud-Mental-inclusion-socialestigma.pdf>, acceso 29 de abril de 2016. 